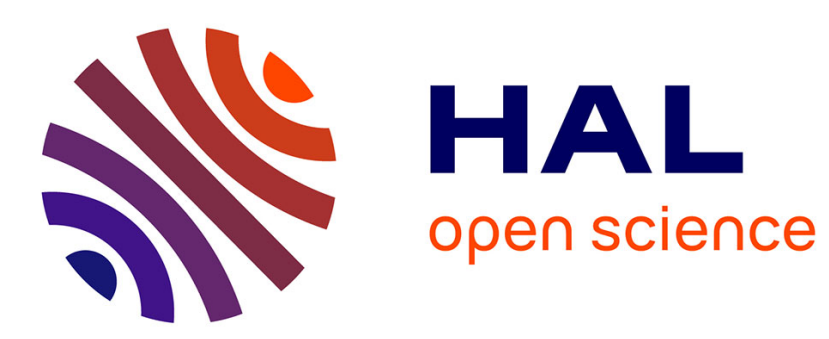

\title{
PME et groupements : antinomie définitive ou nouvelle nécessité?
}

Martine Boutary, Marie-Christine Longe Monnoyer, Raphaëlle Faure

\section{To cite this version:}

Martine Boutary, Marie-Christine Longe Monnoyer, Raphälle Faure. PME et groupements: antinomie définitive ou nouvelle nécessité?. La Revue des Sciences de Gestion, 2012, 10.3917/rsg.257.0101 . hal-02542794

\section{HAL Id: hal-02542794 \\ https://hal.science/hal-02542794}

Submitted on 14 Apr 2020

HAL is a multi-disciplinary open access archive for the deposit and dissemination of scientific research documents, whether they are published or not. The documents may come from teaching and research institutions in France or abroad, or from public or private research centers.
L'archive ouverte pluridisciplinaire HAL, est destinée au dépôt et à la diffusion de documents scientifiques de niveau recherche, publiés ou non, émanant des établissements d'enseignement et de recherche français ou étrangers, des laboratoires publics ou privés. 


\title{
PME et groupements : antinomie définitive ou nouvelle nécessité ?
}

par Martine Boutary, Marie-Christine Monnoyer, Raphaëlle Faure

\begin{abstract}
Martine BOUTARY
Enseignant Chercheur Université de Toulouse Toulouse Business School

France
\end{abstract}

\section{Marie-Christine MONNOYER}

Professeur en sciences de gestion

(IAE Toulouse)

Université Toulouse 1 Sciences Sociales,

France

\section{Raphaëlle FAURE}

Etudiante IEP Toulouse,

Stagiaire Direction Régionale du Commerce Extérieur

France 
Résumé :

PME et groupements : antinomie définitive ou nouvelle nécessité ?

par Martine Boutary, Marie-Christine Monnoyer, Raphaëlle Faure

Dans un contexte de globalisation économique, les groupements d'entreprises peuvent être pour les PME des choix stratégiques pertinents. Combinant flexibilité et dynamisme, ces stratégies de coopération apparaissent comme des modes d'organisation permettant la mutualisation des ressources -remédiant aux manques de moyens structurels des PME- mais aussi des changements organisationnels visant à plus d'efficience.

Pour autant, leur mise en œuvre reste complexe, les caractéristiques de la PME «classique » n'étant pas toujours compatibles avec celles du réseau. Cet article étudie le cas d'un groupement de coopératives viticoles sur ces différents aspects, pour mettre en évidence les obstacles à surmonter pour que les entreprises adhérentes puissent profiter dans les meilleures conditions des apports du groupement.

Mots-clés : PME, groupements, stratégie

\begin{abstract}
:
Groups and SME's : absolute contradiction in terms or new necessity ?

by Martine Boutary, Marie-Christine Monnoyer, Raphaëlle Faure

In a globalisation context, to built a consortium between SMEs seams an efficient strategy. To cooperate is a way to access to a variety of resources, which are lacking generally to SMEs. Such a cooperation allows new organisations which are often more productive.

However to create a consortium is not easy. SMEs are not confident in network organisations. To enlighten the complexity of the management of a consortium, and to give some keys to SMEs, we have chosen to illustrate our analysis with a real case : a consortium of wine co-operatives.
\end{abstract}

Keywords : SMEs, groupings, strategy

\title{
Resumen :
}

Agrupaciones y PYME : antinomia definitiva o una nueva necesidad i

por Evelyne Boutary, Marie-Christine Monnoyer, Raphaëlle Faure

En un contexto de globalización económica, las agrupaciones de empresas pueden ser para las PYME una elección estratégica pertinente. Combinando flexibilidad y dinamismo, estas estrategias de cooperación se identifican como modos de organización que permiten la puesta en común de los recursos -poniendo remedio a la falta de medios estructurales de las PYME- pero también los cambios de organización que apuntan a ser más eficientes.

Por lo tanto su puesta en marcha es compleja, ya que las características de las PYME "clásicas" no son siempre compatibles con las de la red. Este artículo estudia el caso de una agrupación de cooperativas vitícolas sobre estos diferentes aspectos, para subrayar los obstáculos que tendrían que superar para que las empresas afiliadas puedan aprovecharse en las mejores condiciones de las aportaciones de la agrupación.

Palabras claves : Las PYME, agrupaciones, estrategia 


\section{PME et groupement : antinomie définitive ou nouvelle nécessité ?}

Le contexte actuel de très forte ouverture des marchés met les entreprises face à des pressions concurrentielles fortes et des opportunités nouvelles de développement.

Pour celles dont les chercheurs ont souligné la faiblesse des ressources humaines et financières, il est souvent difficile d'engager les investissements nécessaires à la mise en oeuvre de stratégies adéquates. Dans un avis adopté au cours de sa séance du 11 juillet 2007, intitulé PME et Commerce Extérieur, le Conseil Economique et Social met en avant l'évolution préoccupante des exportations françaises et la présence insuffisante des PME. L'une des hypothèses est que la taille de ces entreprises est insuffisante pour se confronter aux enjeux du développement -en particulier international-. Ceci conduit le CES à encourager la création de groupements d'entreprises : "Les avantages de cette forme d'organisation et de coopération entre PME sont parfaitement connus: meilleure répartition des coûts et coordination plus facile de la filière, diversification de l'activité de l'entreprise et contrôle plus aisé de la distribution, réalisation d'économies d'échelles par la mise en place de services communs » (Conseil Économique et Social 2007).

Les groupements de PME sont-ils une «solution » pour saisir des opportunités ?

Ils bénéficient certes d'un présupposé tout à fait positif: réponse à la nécessité d'investissements nécessaires pour rester compétitif sur le marché mondial, opportunité d'innover sur de nouveaux produits ou de nouvelles offres impossibles à générer de façon individuelle. Pour autant, malgré un discours institutionnel très encourageant, le déploiement de groupements de PME reste faible. Les PME seraient-elles imperméables aux promesses des groupements ou plutôt dans l'incapacité de se plier aux contraintes que ces derniers supposent? La création d'un groupement engage chaque entreprise dans une réflexion collective qui a des impacts sur les organisations «individuelles ». Quels sont les obstacles qui se posent à cette coordination collective?

Pour mieux comprendre les difficultés ressenties, nous proposons un retour sur la littérature concernant les spécificités de gestion des PME et celles des réseaux pour appréhender d'une part, ce que ces entreprises peuvent gagner à associer leurs ressources et d'autre part, les réticences qu'elles ont à intégrer ce type de démarche. Puis, nous confronterons le discours théorique à la réalité du terrain en analysant un groupement de 4 caves vinicoles, en termes de construction puis de fonctionnement du groupement, et des influences que celui-ci a sur chacune des caves d'origine. 


\section{PME : un schéma dominé par la gestion de proximité}

Depuis 40 ans, la recherche autour des PME a éclairé plusieurs points : la définition de la PME, de ses contours; les caractéristiques du dirigeant; les caractéristiques des entreprises elles-mêmes puis de leur processus de développement et des stratégies choisies.

Nous retiendrons de ces différents travaux quelques points saillants qui peuvent éclairer la compréhension de notre problématique :

- Le dirigeant de la PME est un personnage capital pour la gestion et le développement de l'entreprise ; «son statut, sa trajectoire sociale et professionnelle jouent un rôle décisif » (Nadine Levratto, 2007) dans la gestion de la PME ; sa personnalité et son expérience sont des éléments forts d'influence : «Il est de plus en plus reconnu que si la PME ne peut pas être approchée comme une grande entreprise en miniature, c'est justement en raison de l'omniprésence, de l'omnipotence et de l'égotrophie dudit dirigeant» (Brahim Allali, 2002). Pierre-André Julien (1997) souligne que le processus de décision du dirigeant de PME entraîne une centralisation de la gestion autour de ce dernier ; à ce phénomène s'ajoute un faible degré de spécialisation, qui conduit le dirigeant à prendre part à (et même à être maître de) l'ensemble des décisions stratégiques mais aussi de points plus opérationnels (c'est par exemple lui qui, dans de nombreuses entreprises, décidera d'aborder un marché mais aussi se déplacera pour rencontrer les clients).

- Les approches classiques soulignent la faiblesse des ressources des PME, souvent marquées par une quasi-pénurie permanente, et une forte soumission à l'environnement (Eric Neilsen, 1974 ; J. Schuman et J. Seeger,1986 ; A. Anderson et M. Atkins, 2001). A contrario, c'est la valorisation de toutes les connaissances, capitale dans des entreprises aux ressources limitées mais aux savoirs souvent très spécifiques, qui permet de se différencier et de rester compétitif (G. Hamel et C.K. Prahalad, 1990). Le groupement est alors considéré comme une autre forme d'organisation, pouvant aboutir à une performance différente, ouvrant de nouvelles perspectives aux PME.

- La préférence est souvent donnée à la gestion de proximité (O. Torres, 2007) : la hiérarchie est très « resserrée » autour du dirigeant, les échelons sont peu nombreux ; la proximité des salariés facilite les contacts réguliers et fréquents entre eux, avec un mode de coordination centré sur l'ajustement mutuel; les systèmes d'information sont simples et informels (parce que le dialogue et la rencontre physique des individus sont fréquents; enfin, la stratégie, souvent qualifiée d'intuitive, d'implicite, répond à une perception forte de la réactivité nécessaire, dans le cadre d'une proximité temporelle toujours orientée vers des 
temps très courts de prise de décision. De là découle un système qui s'appuie sur des informations souvent tacites, peu formalisées, et donc difficilement transmissibles.

L'ensemble de ces caractéristiques peut avoir une influence sur la perception de l'intérêt des groupements et la capacité à travailler avec d'autres entreprises de façon construite, au-delà d'un engagement transactionnel ponctuel. Par exemple, N. Levratto (2007) souligne que «les petites entreprises ne sauraient être conçues comme des entités isolées; elles s'insèrent désormais dans un processus quasi général de réorganisation des chaînes de valeur où la production proprement dite capte une part de plus en plus réduite de la valeur ajoutée de la filière ». Dans un tel contexte, rester seul peut être considéré comme dangereux. Le groupement est-il une réponse à cette problématique ?

\section{PME et réseau: des logiques à organiser}

\subsection{Groupement et réseau}

La coopération interfirmes est un concept large, qui a fait l'objet de nombreux travaux et a donné lieu à de nombreuses qualifications. Pourtant, bien qu'il y ait une littérature sur la coopération entre PME, il n'existe pas un corpus sur les groupements de PME.

«La forme de coopération la plus appropriée pour caractériser le groupement est celle de réseau » (S. Peillon, 2005). Le groupement tel que nous l'étudions est considéré comme «un réseau composé d'au moins deux structures indépendantes sur le plan juridique (et généralement plus), qui s'unissent [...] afin de mener un projet commun nécessitant la coordination de leurs comportements et [...] une dimension formelle, allant de la signature d'un contrat jusqu'à la création d'une entreprise conjointe » (S. Peillon, 2005, p. 108).

C'est ce choix conceptuel que nous retiendrons pour la suite de notre recherche. Ceci nous conduit à approfondir la notion de réseau, pour en comprendre les enjeux et les conditions, et analyser ainsi le cas de groupement que nous présenterons.

\subsection{Les divers intérêts des réseaux}

Les définitions des réseaux sont multiples. Nous retiendrons qu'un réseau est «l'articulation de différentes unités géographiquement éclatées, reliées entre elles de façon plus ou moins formelle et organisées dans l'objectif de répondre à des besoins communs, via une collaboration entre partenaires, des comportements et des moyens de liaison, une stratégie et un management différents de ceux déployés dans une seule et même unité (P. Boulanger 1995). 
C'est aussi un espace où les savoirs et les compétences des partenaires interagissent, selon des logiques collectives, se confrontent et s'enrichissent, pour un avenir commun fondé sur des valeurs partagées, une utilité économique et sociale et un accès à des connaissances étendues (S. Bertezene, 2006). Les discours institutionnels encouragent les groupements en arguant d'une force démultipliée qui permet des résultats économiques meilleurs, ce qui met la performance des entreprises au cœur du débat. Mais se pose aussi la question des interactions entre les membres du groupement, des difficultés de mise en œuvre de l'activité collective. (P. Amans et al, 2006).

À quoi servent les réseaux? Différentes approches tentent d'expliquer leur intérêt: la coopération peut être vue comme une logique de réduction des coûts de transaction (on peut par exemple penser à l'embauche de personnel en commun, ou à des investissements de prospection communs sur une destination commune). Dans ce cas, l'idée du groupement est de mieux rentabiliser des investissements indispensables au développement mais néanmoins trop lourds à supporter pour une entreprise seule. D'autres travaux (J.L. Ravix, 1996 ; P. Amans et al. 2006) mettent en évidence l'intérêt d'une structure en réseau pour un meilleur partage des tâches (division du travail) et une meilleure organisation du travail (coordination). L'idée est alors de ne pas faire plusieurs fois la même chose de façon parallèle, et de créer une organisation économiquement plus intéressante. Enfin un 3ème courant de recherche s'intéresse au travail en réseau comme lieu de mise en commun de compétences, savoirs et informations. Il s'agit alors de mutualiser -à des degrés différents- les savoirs internes à chaque entreprise, de se les approprier, pour ensuite pouvoir transformer et améliorer les processus de décision dans les organisations concernées. Ce courant est très pertinent dans le cadre des recherches sur les PME, puisqu'il est admis que celles-ci ont des ressources plus faibles que les grande entreprises (ce qui justifie la mise en commun), mais qu'elles exploitent des savoir-faire plus spécifiques (PA. Julien et M. Marchesnay, 1996), ce qui justifie l'intérêt de la mise en commun.

\subsection{Réseau et PME : attirances et différences}

Nous avons souligné la spécificité de gestion des PME et relevé les enjeux de la constitution de réseaux. Il nous semble pertinent d'observer plus particulièrement trois points qui semblent ne pas se faire toujours écho, les enjeux pouvant être différents entre la PME et le groupement auquel elle appartient : il s'agit de la volonté d'indépendance des dirigeants, de la gestion de proximité, et enfin du temps pris en compte. 


\subsubsection{Du «un » au « tout » : l’indépendance remise en question}

Travailler en réseau implique une réflexion qui affecte le sentiment d'indépendance des dirigeants des PME engagées dans une telle structuration (K. Messeghem, 2002). Le réseau n'est pas un échange séquentiel, c'est une coopération durable, dans laquelle le dirigeant de PME choisit de s'insérer. Plusieurs auteurs soulignent que l'une des dimensions d'analyse des partenariats est le degré de dépendance de la PME au sein de l'ensemble (D. Puthod 1996). «Dans sa forme aboutie, le concept de réseau semble correspondre au stade ultime de l’interdépendance » (C. Leyronas et O. Torres, 1996).

D'autres auteurs notent que l'organisation en réseau, provoquent des changements dans la gestion de la PME (Boulanger 1990, cité par I. Géniaux, 2003) : «Un réseau implique des comportements, des moyens de liaison, une stratégie et un management différents de ce que requiert une entreprise mono-site ». Le dirigeant n'est plus qu'un «maillon d'un espace de fonctionnement plus partagé ». Le réseau gomme la centralité du dirigeant dans le processus décisionnel. Les PME s'engageant dans un groupement passent d'une organisation indépendante, centralisée autour de la figure du dirigeant, à une organisation plus interdépendante et plus décentralisée. Suivant les objectifs du groupement, la gestion de ces interdépendances sera plutôt contractuelle (dans le cas de groupements dits de similitude) ou plutôt relationnelle (dans le cas de groupements dits complémentaires, ayant pour objectif le développement de l'activité de chaque participant) (S. Peillon, 2005).

Le dirigeant de PME n'étant pas toujours prêt à accepter un développement basé sur l'interdépendance, sa décision de collaborer avec d'autres entreprises est très soumise à son adhésion au projet et à sa pro-activité de façon générale (D. Puthod, 1996).

\subsubsection{De la polyvalence du dirigeant isolé à la spécialisation des ressources du réseau}

Le manque de ressources et le mode d'organisation des PME conduisent souvent à des effectifs réduits. Les cadres de PME doivent donc être généralistes et peuvent occuper plusieurs fonctions dans l'entreprise. Le dirigeant est lui-même souvent l'exemple de cette polyvalence. «On peut considérer la PME comme un tout, où toutes les fonctions sont intégrées ou du moins très fortement reliées, et où le propriétaire dirigeant contrôle tous les aspects, en dirigeant plusieurs fonctions et, pour quelque-unes, en y participant directement » (PA. Julien, 1992). O. Torres (2007) parle de proximité fonctionnelle où «la faible spécialisation des tâches, favorise la polyvalence des travailleurs et les contacts entre eux quelle que soit leur fonction ». 
En se structurant en réseau (dans le cadre d'un groupement pour l'export par exemple), les PME peuvent augmenter leur degré de spécialisation. En effet, le regroupement d'entités différentes va permettre une affectation particulière à chaque entité. La mutualisation des ressources permet de créer un ensemble de taille critique et donc de faciliter la division du travail (A. Paradas, 2007). Suivant les ambitions affichées du partenariat, les nouveaux besoins en compétence prennent la forme d'embauche ou d'externalisation. «Le réseau peut être un mode d'organisation permettant de combiner des compétences diverses. On peut même considérer qu'une des raisons d'être du réseau est la complémentarité des compétences et donc la spécialisation des activités entre les différents membres »(O. Torres, 1997).

\subsubsection{Proximité temporelle : le temps court des PME, le temps long de la planification du réseau}

Les dirigeants de PME valorisent la réactivité et la flexibilité pour le client, s'inscrivant ainsi dans un temps «court». Cette proximité temporelle (O. Torres, 2007) dans le management de la PME se traduit par un processus décisionnel plutôt intuitif, rapide et une stratégie avant tout implicite (P. Legoherel et al., 2003): «Ces caractéristiques spécifiques aux petites entreprises constituent des avantages qui leur sont propres, tels la rapidité avec laquelle les décisions sont exécutées, la proximité des marchés, ainsi qu'une grande capacité de s'adapter et de changer d'orientation à court terme » (S. Blili et L. Raymond, 1998)

La création du consortium passe généralement par un business plan détaillé énumérant les objectifs et la stratégie pour les atteindre. Cette définition stratégique, destinée notamment à rassurer ou à convaincre des partenaires financiers privés ou publics, implique un effort de formalisation et de communication. Ceci peut modifier les modalités de gestion de la PME, la faisant passer d'un mode de gestion à court terme, informel, implicite, réactif et intuitif, à un mode plus formel, explicite, proactif avec une vision à plus long terme.

\section{Le cas VINOVALIE : construction d'un groupement de caves coopératives vinicoles}

\subsection{Point méthodologique}

Le choix de la méthode d'étude de cas se justifie dans cette recherche par le fait que nous cherchons à comprendre les modalités de construction du groupement, plus que le résultat luimême. Il importe donc de recueillir des données qualitatives, autorisant la représentation du processus de construction, au travers des représentations subjectives de différents acteurs. E. Lazéga, 1994, souligne que l'analyse des réseaux n'a souvent de sens que dans la mesure 
où une analyse qualitative est faite, permettant une réelle connaissance du contexte, et autorisant une bonne compréhension et interprétation des résultats obtenus malgré le caractère difficilement mesurable du réseau (C. Leyronas, 1996). Elle apparaît alors comme la seule capable d'accroître l'aptitude du chercheur à décrire un système social complexe (C. Marshall et G. Rossman 1989 in P. Amans et al., 2006).

Le choix de l'étude d'un cas unique peut interpeller le lecteur. M. Hlady-Rispal (2002) justifie ce choix notamment dans le cas où le chercheur souhaite révéler un phénomène, non exceptionnel mais qui n'est pas encore accessible à la communauté scientifique. Le cas de la création des groupements d'entreprises reste encore peu étudié, et mérite ce regard exploratoire. M. Hlady Rispal souligne que tout phénomène observé au travers d'un cas unique a potentiellement une portée générale.

La question de la triangulation des données a été prise en compte par la réalisation d'interviews avec des acteurs de structures différentes et ce, dans des lieux différents. Nous nous sommes rendues dans quatre caves différentes, et avons interrogé en face à face, à partir d'un guide d'entretien, le directeur du groupement, les directeurs de deux caves appartenant au groupement, et deux responsables export du groupement, appartenant eux aussi auparavant à deux caves différentes. Ces entretiens ont été enregistrés et retranscrits, pour ensuite être le support d'une analyse par catégorisation.

Un dernier choix consiste à étudier un groupement d'entreprises coopératives qui sont peutêtre un cas particulier de PME. Différents critères nous ont laissé admettre que les coopératives étudiées avaient des points communs avec des PME : leur taille, un très fort ancrage dans une logique territoriale, un centrage sur la notion de «métier », un rapport de proximité avec les différents acteurs (internes et externes à l'entreprise) et au-delà, un mode de gestion de proximité. En revanche, nous admettons que certaines caractéristiques les distinguent de certaines PME : le directeur des caves a une délégation de gestion de la part d'un président élu et de son conseil d'administration chargés de gérer les intérêts ambivalents d'adhérents qui sont à la fois actionnaires et fournisseurs de la cave coopérative. Nous intègrerons ces particularités dans nos analyses.

\subsection{Présentation du cas}

Vinovalie est une Union de caves coopératives viticoles créée en mai 2006. Les caves de Técou et de Rabastens dans le Gaillac, celles de Fronton et des Côtes d'Olt (Cahors) ont regroupé leurs forces pour faire face à un contexte économique de plus en plus difficile. 
En 2001, temps fort d'une première crise dans le secteur du vin, les vins régionaux sont frappés de plein fouet par une baisse de la demande. Les facteurs de la crise sont externes (globalisation du marché, pression concurrentielle, nouvelles habitudes de consommation) et internes (absence de travail sur un processus de différenciation, vision orientée produit, plus que marché). Après un répit en 2003 dû à une crise de production, la demande s'effondre à nouveau en 2004 et 2005 . Pour la $1^{\text {ère }}$ fois dans 1'histoire de la viticulture française, la demande mondiale augmente et les caves françaises vendent moins.

Face à cette situation, quelques-unes d'entre elles décident de tenter un effort de regroupement : «Sans la crise, nous n'aurions peut-être pas dépassé notre individualisme » (Directeur 1).

«Sans la crise, il n'aurait pas été possible d'aller si vite et si loin» (Directeur du groupement).

Le premier objectif du groupement est le développement des ventes sur les marchés extérieurs pour compenser l'affaissement et la pression concurrentielle du marché domestique ${ }^{1}$. Le constat est opérationnel mais aussi stratégique; il fallait réduire les «gaspillages » (les responsables export de deux coopératives s'étaient retrouvés dans le même avion pour aller au Japon dans le cadre d'une mission organisée par la Chambre de Commerce, ils avaient rencontré les mêmes importateurs et en avaient retiré les mêmes conclusions) et il fallait organiser la stratégie pour éviter les actions dispersées et sans visibilité à cause d'une trop faible taille.

Le groupement est créé et immédiatement très «formalisé ${ }^{2}{ }^{2}$ 《 Dans un projet pareil, le point capital c'est la gouvernance; dans un secteur comme celui de l'agriculture, il faut sortir des habitudes et des querelles existantes et inventer de nouvelles organisations; un organigramme, ça sert à éviter les conflits de personnes, c'est très important » (Directeur Vinovalie » Le schéma suivant montre l'organigramme d'une cave (Figure 1) :

\footnotetext{
${ }^{1}$ «Vinovalie, du vin en terre d'Ovalie », Le trait d'union paysan, 23 février 2007.

${ }^{2}$ On ne peut ignorer l'importance dans le processus de l'intervention d'un homme, alors directeur du Syndicat interprofessionnel des viticulteurs du Sud-Ouest, et homme moteur du GIE «Les vignerons du Sud-Ouest ». Ce statut, lui conférant une excellente connaissance du secteur du vin et de ses acteurs régionaux (en plus d'une formation $\mathrm{Bac}+5$ en management), le rend très crédible auprès des directeurs de caves qui le côtoient depuis plusieurs années et lui accordent une très grande confiance. Il devient alors le pilier du groupement, et en sera le directeur.
} 


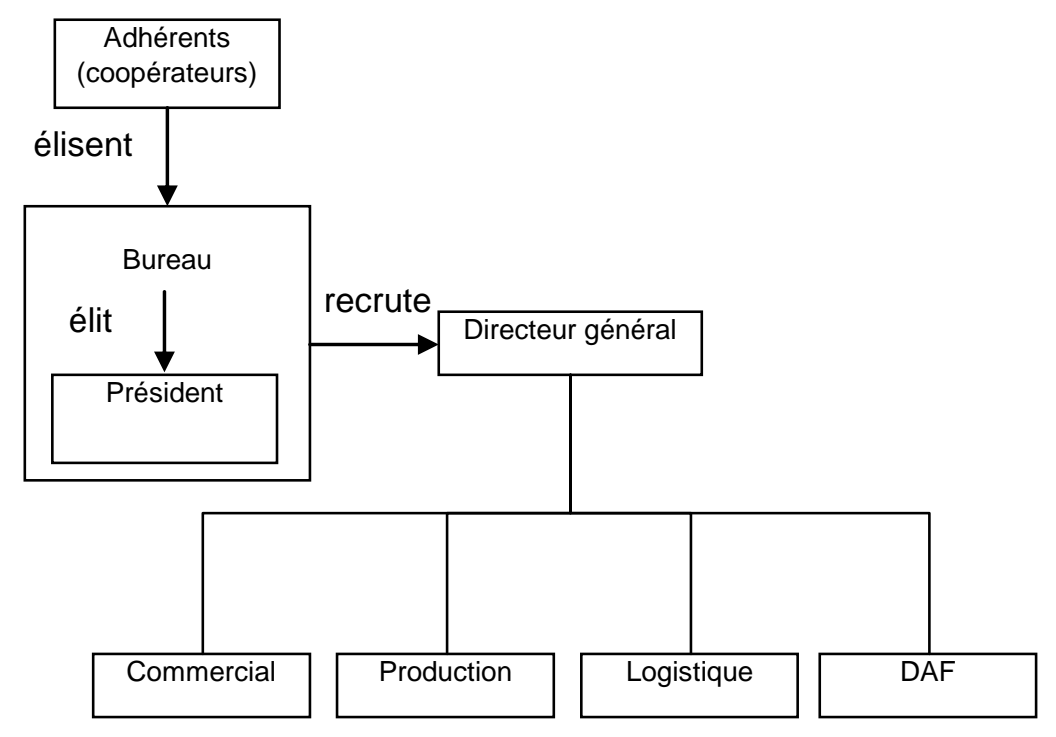

Fig 1 : Organisation générique d'une cave coopérative

Lors de la création de Vinovalie, un Conseil d'administration de 20 personnes est mis en place : 4 membres du bureau de chaque cave ainsi que les 4 présidents de cave. La présidence du groupement est élue, il s'agit d'une des caves (la présidence n'est pas nominative) par ce Conseil d'Administration. Le directeur général du groupement devient le directeur général de toutes les caves, ce qui modifie considérablement la position initiale de chaque directeur de cave. Les responsabilités des uns et des autres changent : par exemple, le directeur des caves d'Olt prend la direction des Caves d'Olt et de Fronton, et se spécialise sur les achats et l'export. C'est lui qui devient le responsable du développement international du groupement, grâce à ses compétences. Le directeur de la cave de Fronton devient responsable de la commercialisation, du marketing et des ventes en vrac ; un autre directeur de caves prend la direction des caves de Rabastens et de Tecou, et la responsabilité du processus qualité pour l'ensemble du groupement. Conscient des changements engendrés par le passage d'une structure de petite dimension à celle d'un groupe plus important, le groupement a embauché un spécialiste en organisation (qui a travaillé dans des grands groupes comme coca-cola). La nouvelle organisation se présente de la façon suivante : 


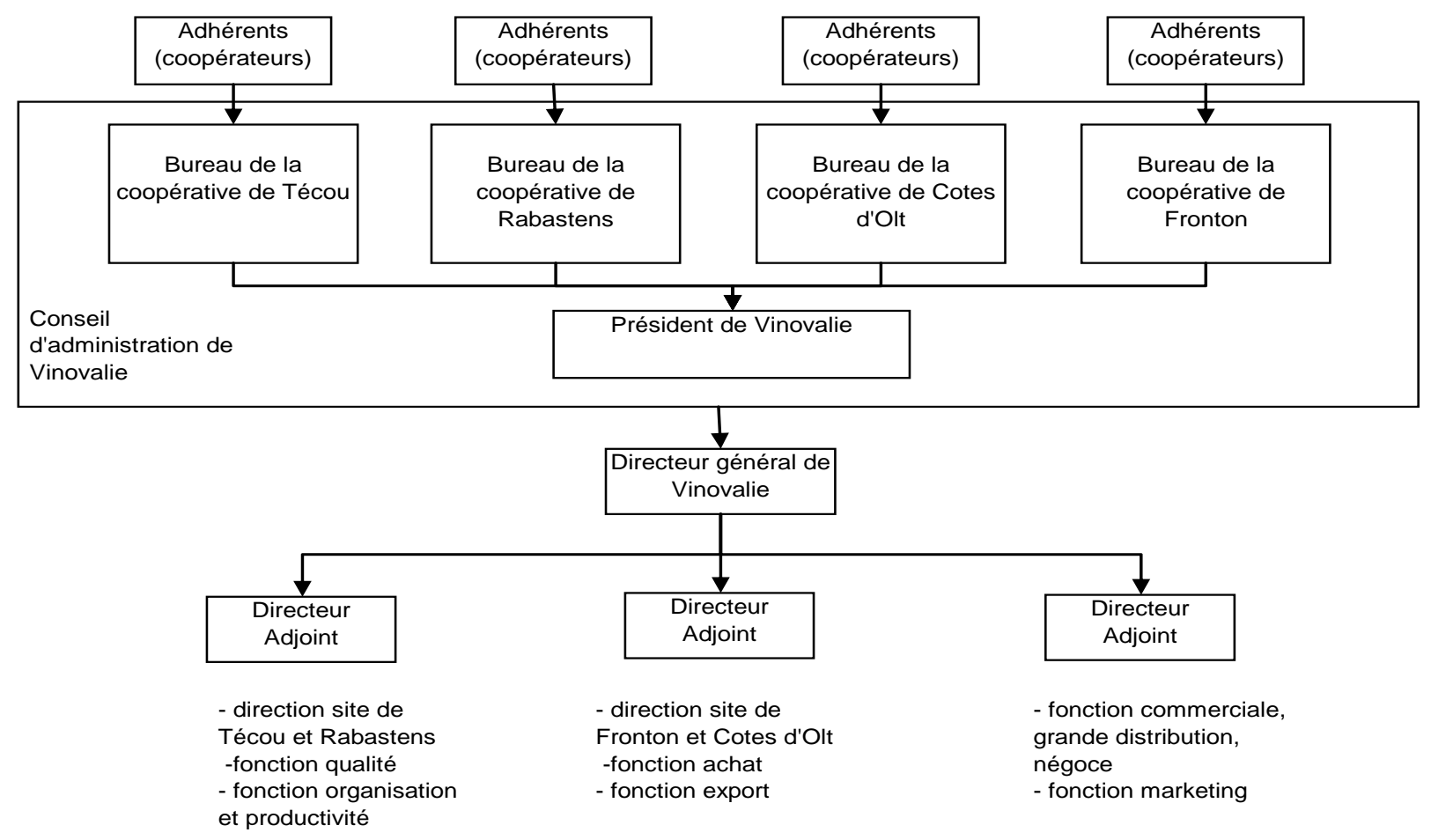

Fig. 2 : Organisation de VINOVALIE

\subsection{Analyse}

\subsubsection{Des éléments de contexte importants et favorables}

La création du réseau a bénéficié de la conjonction de deux éléments :

- d'une part, une pression externe forte et suffisante pour créer un sentiment d'incertitude voire d'affolement conduisant à la remise en cause des existants de chaque coopérative, tant sur le plan de la structure que des stratégies individuelles. Le directeur du groupement qualifie de «chance » la force « du danger environnemental »: un environnement un peu plus favorable n'aurait sans doute pas permis aux caves concernées de se donner les moyens d'une action aussi énergique, malgré l'attitude tout à fait volontariste des directeurs de cave.

- d'autre part, l'existence en interne de relations de confiance et l'émergence d'une tête de réseau reconnue par tous, tant sur le plan des compétences professionnelles que sur le plan humain. Lorsque naît l'idée d'un regroupement (issue notamment d'une analyse stratégique du secteur pointant la taille critique insuffisante des structures de production et l'éclatement de l'offre), la confiance des uns envers les autres va être un support important de la mise en œuvre et de la rapidité des opérations (E. Loubaresse, 2008). 


\subsubsection{Rupture avec l'existant}

Mais si la pression environnementale a été nécessaire pour "penser réseau », c'est parce que les dirigeants ont accepté de «penser autrement » et d'engager une démarche très proactive que le réseau a vu le jour sous une forme très structurée. Ces dirigeants ont immédiatement compris qu'il n'était pas possible de faire quelque chose de façon collective sans toucher les organisations existantes. Il y a donc eu une réflexion stratégique. Du «court termisme » de chaque cave, on passe à une stratégie de long terme d'une organisation collective : «Auparavant, sur chaque cave, il n'y avait pas vraiment de formalisation des objectifs, pas de chiffrage quantitatif ... Vinovalie nous a permis d'être beaucoup plus structurés et d'avoir une vision sur trois ans » (Directeur 1).

La préoccupation «métier », celle attachée aux phases de production du vin et de sa mise en bouteille, n'est plus le seul guide de gestion: "Il faut assurer un revenu par métier et spécialiser les métiers dans une logique de process industriel, source d'économie d'échelle et de professionnalisme ».

Le changement important que nous permet de constater l'évolution des organigrammes avant et après création du groupement souligne une stratégie très volontariste, avec une notion de « rupture des organisations existantes » au moment du démarrage du groupement. Pour autant, la construction du groupement s'inscrit dans une dynamique et dans le temps. L'organisation en réseau se réfléchit et s'invente dans un but de progrès permanent (L. Saglietto, 1997). Initialement créé pour des considérations commerciales, Vinovalie a ensuite permis une réflexion sur un processus de réorganisation (services commerciaux, mutualisation des achats) ayant pour finalité la réduction des coûts. Parallèllement, la mise en commun d'un système d'information pour l'ensemble des coopératives a autorisé la création d'indicateurs communs, permettant une mesure explicite de la performance.

Ce nouveau mode d'organisation abaisse considérablement la centralité du dirigeant de chaque coopérative, qui ne décide plus seul de la stratégie à suivre. L'interdépendance se construit au fur et à mesure, comme outil au service de la stratégie élaborée. C'est le dirigeant du groupement qui en est le maître d'œuvre, «l'architecte réseau », autour d'un travail de rationalisation et d'optimisation des structures existantes, dans une logique qui passe du court terme au long terme et à une vision globale. Certaines étapes ont été franchies (la réorganisation commerciale, le réaménagement de l'organisation de direction, la propriété des marques). D'autres sont en cours (aménagement d'un centre logistique). Une dernière étape reste à venir, celle qui, de l'avis du directeur du groupement, est capitale : la mise en place 
d'un site d'embouteillage commun. On touche là, en effet, au cœur de métier, et au cœur des acteurs. On touche le produit, l'objet de fierté, la raison d'être de la coopérative et c'est difficile. Il faudra là beaucoup de savoir-faire pour convaincre, obliger, écouter, orienter sans se départir de cet objectif stratégique énoncé. Là encore, la contrainte externe crée une condition favorable : la logistique devient de plus en plus chère et ne peut supporter de rester dans des situations historiques d'éclatement géographique.

Le nouveau système bénéficie des synergies créées : la constitution du service export et la spécialisation des commerciaux permettent d'optimiser les déplacements, d'approfondir le travail de prospection de chacun et de tisser des liens plus personnels et plus stables avec la clientèle étrangère. La taille critique atteinte par le groupement a généré l'attention nouvelle d'agents jusqu'alors peu intéressés par une offre insuffisante, tant sur le plan de la diversité des gammes que sur le plan des quantités offertes.

\subsubsection{Vers une spécialisation des fonctions}

En se regroupant, les quatre coopératives ont créé un service commercial commun. La logique des réseaux évoquée par la littérature, concernant le partage des tâches et la spécialisation, semble ici s'appliquer: il y a modification de la nature de chaque coopérative par la réaffectation de chaque personne à une fonction centrée sur ses compétences. Les employés de chaque service commercial des quatre caves viticoles, sont devenus salariés de la nouvelle structure Vinovalie. Les échanges sont plus nombreux et la concertation plus grande : «...les commerciaux de Vinovalie vendent les choix commerciaux aux directeurs de chaque cave et inversement les directeurs vendent leurs idées et leurs produits à Vinovalie ». Les directeurs de cave sont passés de «supérieur hiérarchique direct » à «fournisseur interne » auprès des commerciaux : "Chaque directeur a un rôle à jouer pour inciter les commerciaux de Vinovalie à vendre sa gamme. Ils doivent presque nous vendre le produit, en interne. » 
Jusqu'à présent, étant donné la jeunesse de l'Union, aucune nouvelle embauche n'a été faite. Mais des besoins en compétences spécifiques se font sentir, comme par exemple en marketing

\begin{tabular}{|l|l|l|}
\hline & Avant la création de l'Union & 1 an après la création de l'Union \\
\hline Structure : & $\begin{array}{l}4 \text { caves coopératives } \\
4 \text { directeurs de cave } \\
3 \text { services export (absent à Técou), } \\
4 \text { personnes }\end{array}$ & $\begin{array}{l}4 \text { caves coopératives } \\
\text { structure commerciale chapotant } \\
\text { 1'ensemble } \\
1 \text { service export, 4 personnes }\end{array}$ \\
\hline Exportations & 3 caves sur les quatre exportent & $\begin{array}{l}\text { En augmentation de près de 20\% } \\
\text { en une année sur l'ensemble des } \\
\text { caves. } \\
\text { Toutes les caves exportent }\end{array}$ \\
\hline $\begin{array}{l}\text { Niveau de } \\
\text { centralisation de la } \\
\text { décision }\end{array}$ & $\begin{array}{l}\text { Niveau de centralisation mesuré } \\
\text { dans chaque cave (tradition } \\
\text { coopérative) }\end{array}$ & $\begin{array}{l}\text { Tendance à plus de partage du } \\
\text { pouvoir de décision ; création de } \\
\text { fonctions transversale (logistique, } \\
\text { qualité, export...) }\end{array}$ \\
\hline Spécialisation & $\begin{array}{l}\text { Polyvalence importante au sein des } \\
\text { différentes caves }\end{array}$ & $\begin{array}{l}\text { Tendance à la spécialisation des } \\
\text { tâches (services commerciaux) - } \\
\text { tendance à l'externalisation }\end{array}$ \\
\hline
\end{tabular}

ou pour le développement des exportations. Antérieurement, trois caves sur quatre avaient une personne en charge (prospection et gestion) des marchés étrangers. Le service export de Vinovalie est désormais composé de 4 personnes, dont les trois responsables export existants, qui se sont spécialisés sur une zone géographique, en fonction de leurs expériences.

La spécialisation s'est aussi portée sur l'organisation de la production de chaque cave. Ainsi, à titre d'exemple, la cave de Fronton, mieux équipée pour la production de vin rosé, a récupéré l'assemblage de ces types de vin de l'ensemble du groupement pour augmenter l'efficience de la production.

Depuis la création de Vinovalie, le recours à l'externalisation est plus fréquent. Alors que l'on demandait aux employés des caves coopératives d'être polyvalents, de se débrouiller avec des moyens restreints, les salariés de Vinovalie sont plus concentrés sur leurs missions. Cette focalisation se traduit par l'utilisation de spécialistes externes à l'organisation. Par exemple, pour la communication, Vinovalie passe par des designers professionnels, des graphistes et des traducteurs, pour se concentrer sur son métier : «Vinovalie c'est plus gros, donc plus de moyens ... avant je faisais les traductions moi-même parce que je me débrouille en anglais.... Maintenant on passe par une société de service. Le faire soi-même, ça prend des heures, on n'est pas professionnel de la traduction, on n'est pas très productif ... »

\subsubsection{De l'exportation des coopératives à l'internationalisation du groupement}

Dès sa création, le directeur du groupement a affiché des objectifs forts pour le développement des exportations : + 30\% par an à l'export pendant 3 ans. La raison est simple : d'une part, c'est à l'export que les marges de croissance sont les plus importantes ; 
d'autre part, l'activité export est très faible : sur 30M d'euros de CA pour l'ensemble des caves réunies, $2 \mathrm{M}$ sont issus de ventes hors frontières : «on était très mauvais, certains encore plus que d'autres ». L'attitude des dirigeants de cette cave face à l'exportation était plutôt passive (par choix stratégique dû à la faiblesse de la taille, méconnaissance des marchés extérieurs et des procédures d'export). Le groupement Vinovalie a permis de «desserrer le frein » que pouvait constituer leur attitude.

Pour éviter les «doublons», et mieux organiser les ressources, une réorganisation des marchés a été effectuée, les forces ont été recentrées : un seul importateur (le plus important) a remplacé les 3 précédents au Québec ; (il a donc l'avantage de volumes plus importants ce qui lui permet de faire des efforts de commercialisation plus visibles), 2 agents seulement ont été conservés en Grande-Bretagne.

Mais la pensée stratégique ne s'est pas arrêtée à la seule exportation. La réflexion s'est tournée vers la prise en compte de marchés jugés stratégiques mais inaccessibles aux moyens du seul groupement. Le marché chinois fait partie de ces exemples. Au vu des fortes barrières à l'entrée de ce marché, Vinovalie s'est associé à un autre groupement de caves françaises pour créer une Joint-Venture avec un partenaire chinois. Chaque union a investi $40000 €$ dans ce projet, et la prospection est déjà lancée, parallèlement à l'ouverture d'un magasin à Shangaï (l'idée est d'ouvrir 2 autres magasins à Pékin et Canton). L'objectif est de vendre 50000 bouteilles dans 2 ans (pour repère, le groupement qui a servi de «modèle » à Vinovalie en vend à ce jour 200000 après 15 ans de présence).

Le même objectif est actuellement à l'étude pour un développement aux États-Unis.

D'une exportation opportuniste caractérisant souvent les entreprises exportatrices «sans stratégie », le groupement est passé à la mise en place d'objectifs de développement à long terme. La formalisation stratégique a montré l'intérêt de certains marchés, inaccessibles sans partenariat plus élaboré. La structuration financière forte du groupement, avec notamment la mise en place de capitaux propres importants, a permis d'envisager un développement de plus grande ampleur. De nouveaux indicateurs de performance export sont venus compléter celui du chiffre d'affaires «export», en valeur absolue et en pourcentage du chiffre d'affaires global; par exemple sont évalués la qualité des agents importateurs (et notamment leur importance sur le marché représenté, et l'investissement fait pour développer les gammes) ; la mise en place des accords de partenariat (celui en cours de construction sur la Chine et celui en projet sur les US).

$\mathrm{Du}$ développement des exportations, l'action s'est alors orientée vers un développement international et la prise de participation dans une structure franco-chinoise. On constate 
l'apparition d'une coopérative globale, organisée au-delà de la gestion de proximité des entreprises seulement exportatrices: la planification prend le pas sur l'organisation opportuniste et à court terme, l'organisation et la spécialisation supplantent la polyvalence des acteurs, l'interdépendance est revendiquée, construite contre l'isolement pour améliorer les performances, en termes d'efficience comme d'efficacité. L'espace de marchés est étendu, mais aussi l'espace de fonctionnement des entreprises. De tels résultats n'incitent pas à revenir en arrière.

\section{Conclusion :}

Suivant les objectifs du groupement d'entreprises, les avantages peuvent varier : la mise en commun des ressources humaines, financières ou informationnelles, le partage des fonctions ou des investissements, ou l'abaissement des coûts de gestion de chaque entreprise adhérente. Les entreprises peuvent aussi se regrouper pour développer de nouveaux produits et/ou de nouveaux marchés. Il s'agit alors de créer des synergies et de déployer des moyens non mobilisables de façon individuelle, revenant de cette façon sur l'insuffisance de ressources des PME. Dans les deux cas de figure, les chercheurs, comme les institutionnels ou les entreprises, s'accordent à penser que l'intérêt est bien là et que grâce au regroupement, de nouvelles conditions de performance ou des nouveaux projets peuvent émerger et rendre les entreprises plus compétitives.

Mais l'atteinte de ces objectifs ne se fait pas sans efforts. Le fait pour une entreprise d'adhérer à un groupement n'est pas neutre. L'indépendance si souvent décrite des dirigeants de PME est plus ou moins remise en question. Le centre de gravité de l'entreprise se déplace, avec celui de son dirigeant. L'appartenance à un groupement, notamment quand il est question de développer de nouvelles activités ou de nouveaux marchés, va s'accompagner de modifications organisationnelles importantes. La gestion centralisée, la polyvalence des acteurs (dirigeants et autres), la préférence donnée à un fonctionnement à court terme, sont remises en question au profit d'organisations plus formalisées, plus planifiées, plus interdépendantes. Paradoxalement, il semble que l'environnement international, tant par le haut niveau de contraintes diverses qu'il impose que par les opportunités qu'il représente et qui sont inaccessibles à des entreprises insuffisamment dotées de ressources, soit un élément externe particulièrement incitatif. Dans le cas que nous avons étudié, nous montrons à quel point la contrainte concurrentielle, au-delà des difficultés qu'elle génère dans les entreprises, peut être à l'origine d'une nouvelle organisation autour d'objectifs ambitieux. 
Un changement de mode de gestion - passant d'une proximité hiérarchique, fonctionnelle et temporelle à une gestion «à distance »-, est un défi que ne souhaitent ou ne peuvent relever tous les dirigeants de PME. Il est tout d'abord nécessaire de cadrer l'objectif : économies d'échelle ou croissance? Si un contrat simple peut permettre de gérer assez simplement la mise en commun de certaines ressources pour faire quelques économies, il n'est en revanche pas facile de créer et de pérenniser des groupements dont l'objectif est le développement d'affaires pour chaque entreprise adhérente. Pour éviter des risques d'opportunisme individuel, ce type de groupement nécessite une gestion contractuelle, mais aussi des relations de confiance, souvent construites grâce à des expériences communes antérieures à la création du groupement.

Il est donc difficile de créer des groupements «ex-nihilo». L'analyse que nous venons de présenter incite à penser, notamment de la part des Pouvoirs Publics, à l'animation -et peutêtre au financement- de temps intermédiaires, pendant lesquels les entreprises apprennent à cadrer leurs objectifs communs et un mode de fonctionnement. Il est nécessaire d'intégrer la planification, la délégation, l'organisation pour que les projets puissent être déployés. Les pistes de recherche sont nombreuses sur ces sujets, encore insuffisamment explorés.

Notre travail a des limites, portant notamment sur la question de la similitude entre coopératives et PME. Nous avons posé cette hypothèse de similitude, tout en soulignant quelques caractéristiques différenciatrices. Nous avons justifié ce choix méthodologique, et pensons qu'il est possible d'utiliser ces travaux pour mieux comprendre les facteurs de succès et d'échec des groupements de PME à l'export, si difficiles à promouvoir. Mais il serait pertinent d'étudier la structuration d'autres groupements de PME indépendantes et non coopératives pour étendre la validité de ce travail. Il serait aussi intéressant d'observer dans le temps l'évolution du groupement ici étudié, pour valider la pérennité des actions mises en place.

Enfin, il serait sans doute utile que les discours encourageant la création de réseaux insistent d'une part, sur la diversité des apports possibles à chaque entreprise participante, d'autre part, sur les conditions de réussite de ces nouvelles organisations. Mettre en évidence, de façon positive les apports de la formalisation, de la planification, de l'interdépendance (pourtant contraires à l'idée « traditionnelle de la PME ») pourrait ouvrir de nouvelles perspectives pour des dirigeants qui valorisent souvent la réactivité et l'informel comme arguments majeurs et différenciateurs de leur réussite. 


\section{Bibliographie :}

Allali B. (2007), «PME et internationalisation», Management des PME, de la création à la croissance, sous la direction de Jacques Filion, édition du Renouveau Pédagogique Inc. 2007, p. 335 à 347

Amabile S. et Gadille M., 2006, Coopération interentreprises, systèmes d'information et renouvellement de l'attention organisationnelle, AIMS , Annecy, Juin

Amans P., Ayed Zambaa F., Descargues R., Loup S., Acquis et perspectives de la compréhension des relations entre PME : L'analyse d'un cas par le concept de réseau clignotant ; 8ème CIFEPME, Montpellier 2006

Anderson, A. et Atkins, M. (2001). Business Strategies for Entrepreneurial Small Firms.Strategic Change 10 (6): 311-324

Bertezene S., 2006, Quelles sont les conditions de succès d'un réseau? Le cas des réseaux gérontologiques, AIMS, Annecy, Juin

Biardeau S., Bourcieu S., Salgado M., (2003), «Conséquences du volontarisme stratégique sur le développement international des PME », Colloque l'entrepreneur en action : contextes et pratiques, octobre 2003.

Blili S. et Raymond L., (1998), "Les systèmes d'information", p. 221-243 dans PME : bilan et perspectives, Editions Economica, deuxième édition, 351p.

Boulanger P., 1995, Organiser l'entreprise en réseau-la pyramide éclatée, Paris, Ed. Nathan

Bourcieu S., 2000, L'internationalisation des PME: du déterminisme au volontarisme stratégique, Gestion 2 (1) p. 12-22

Boutary M. (2007), «Technologies de l'information et de la communication dans les PME internationalisées », Management des PME, de la création à la croissance, sous la direction de Jacques Filion, édition du Renouveau Pédagogique Inc. 2007, p. 321 à 333.

Cabrol M., (2007), "Les premières phases de l'internationalisation des entreprises nouvellement créées", 16 ième Conférence Internationale de Management Stratégique

CES (2007), «PME et commerce extérieur», Avis et rapport du Conseil Economique et Social, présenté par Jean François Roubaud, juillet 2007. .

Chassagon V., (2008) «Qu'est-ce qu'une firme réseau », Congrès de l'AIMS, Sophia Antipolis

Gankema, H.G., H.R. Snuif et P.S. Zwart (2000), "The internationalization process of small and medium-sized enterprises: An evaluation of stage theory", Journal of Small Business Management, Vol. 38, n 4 , p. 15-27.

Géniaux I., Mira-Bonnardel S. (2003), «Le réseau d'entreprises : aboutissement d'une trajectoire organisationnelle ou forme transitoire d'organisation», $12^{\text {ième }}$ Conférence de l'Association Internationale de Management Stratégique.

Hamel G., Prahalad C.K. (1990), The Core Competence of the Corporation, Harvard Business Review, $n^{\circ} 68$,

Hermann Simon (1998), «Les champions cachés de la performance », Edition Dunod 1998.

Hlady Rispal M., 2002, "La méthode des cas, Application à la recherche en gestion », De Boeck Université

Julien P.A. (1996), Joyal, A., P.-A. Julien, L. Deshaies et C. Ramangalahy, « Typologie des comportements stratégiques des PME exportatrices », Revue Gestion, Vol. 21, no 1, 1996, pages 2937.

Julien P.A. (1997), «Les PME, bilan et perspectives », Economica

Julien PA et Marchesnay M. (1996), L'entrepreneuriat, Economica

Julien.P.A et Marchesnay M., (1992), "Des procédures aux processus stratégiques dans les PME", pp 97-129 dans "Perspectives en management stratégique", Tome 1:1992/1993, sous la direction d'A.Noël, Editions Economica-Gestion, 431p. 
Khayat, I., (2004), "L'internationalisation des PME : vers une approche intégrative", 7ieme CIFEPME Laghzaoui S., (2007), "Ressources et compétences : une nouvelle grille de lecture de l'internationalisation des PME", $16{ }^{\text {ième }}$ Conférence Internationale de Management Stratégique

Lazega Emmanuel, Analyse de réseaux et sociologie des organisations, Revue française de sociologie, Année 1994, Volume 35 , n 35-2, pp. 293-320

Legoherel P, Callot P., Gallopel K., Peters M., (2003), Dimensions psychologiques, processus de prise de decision et attitude envers le risqué : une étude des dirigeants de PME, La Revue des Sciences de Gestion, $\mathrm{n}^{\circ} 199, \mathrm{p} .51$

Levratto N., (2007), Particularités de la PME, in Management des PME, sous la direction de J.L. Filhon,

Leyronas C., Torres O. (1996), «Stratégie de mondialisation et PME : l'instruction d'un paradoxe », $3^{\text {ième }}$ Congrès International Francophone de le PME, Québec, Canada, 25-27 juin.

Loubaresse E., (2008) «Qui pilote les réseaux interorganisationnels ? Caractéristiques des «brokers » de réseaux locaux d'organisations", Congrès de l'Association Internationale de Management Stratégique, Mai

Messeghem K. (2002) Peut-on concilier logiques managériale et entrepreneuriale en PME ? La revue des Sciences de Gestion, No 194, P. 35

Neilsen, E. (1974). Contingency Theory Applied to Small Business Organizations, Human Relations 27(4): 357-379

Pacitto J.C., (2006), «L'internationalisation des PME: une tentative d'état des lieux », 8ième CIFEPME

Paradas A., (2007), Mutualiser la formation et le recrutement dans les PME : une variété de réponses, La Revue des Sciences de Gestion, No 226 -227, p. 147

Peillon S., (2005), «Une analyse dynamique du pilotage des PME », Revue Internationale PME, Vol.18, No 1

Puthod D. (1999), «L'alliance, une option permettant de contourner les dilemmes classiques de la $P M E »$, PME de nouvelles approches, édition Economica, 1999

Puthod D., Ganassali S. (1996), "Comprendre les alliances de PME (à partir d'une base de

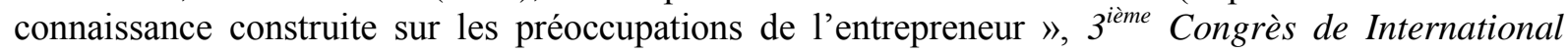
Francophone sur la PME.

Ravix J.L., "Un concept de réseau pour analyser l'organisation industrielle", Chapitre de l'ouvrage Coopération entre les entreprises et organisation industrielle, sous la direction de J.L. Ravix, coll. Recherche et Entreprise : éd. du CNRS, sept 1996.

Rorive B. (2003), «L'entreprise réseau : unicité de la forme, diversité des situations », $14^{\text {ième }}$ Congrès de l'AGRH, Grenoble, novembre 2003

Saglietto L., (1997), Communication et apprentissage dans les Joint Ventures internationales, La Revue des Sciences de gestion, No 164-165, p. 153

Schuman, J. et Seeger, J. (1986). The Theory and Practice of Strategic Management in Smaller Rapid Growth Companies, American Journal of Small Business 11(1): 7-18

Torres O. (2007), «Approche descriptive de la spécificité de gestion des PME : le mix de proximité », Management des PME, de la création à la croissance, sous la direction de Jacques Filion, édition du Renouveau Pédagogique Inc. 2007, p. 23 à 33

Torres O., (1997), «Territoire, PME globales et réseaux transnationaux », dans «Globalisation et compétitivité », F. Sifrioui, travaux et recherche du réseau ESPRIT, p81-98. 